\title{
The boundary integral method for the steady rotating Navier-Stokes equations in exterior domain (I): the existence of solution
}

\author{
Rong An and Kaitai Li
}

\begin{abstract}
In this paper, we apply the boundary integral method to the steady rotating Navier-Stokes equations in exterior domain. Introducing some open ball which decomposes the exterior domain into a finite domain and a infinite domain, we obtain a coupled problem by the steady rotating Navier-Stokes equations in finite domain and a boundary integral equation without using the artificial boundary condition. For the coupled problem, we show the existence of solution in a convex set.
\end{abstract}

Mathematics Subject Classification (2000). 76M15, 76U05.

Keywords. Rotating Navier-Stokes equations, Exterior domain, Boundary integral method.

\section{Introduction}

Let $\Omega^{c} \subset \mathbb{R}^{3}$ be a simply connected bounded domain with smooth boundary $\Gamma$. Assume that $\Omega^{c}$ is rotating with the constant angular velocity $\vec{\omega}$ in a viscous incompressible fluid filling the entire space, then the rotating Navier-Stokes equations for the fluid occupying $\Omega=\mathbb{R}^{3} \backslash \overline{\Omega^{c}}$ consist in finding the velocity vector $u$ for the fluid and the pressure $p$ such that

$$
\begin{cases}\frac{\partial u}{\partial t}-\nu \Delta u+u \cdot \nabla u-(\vec{\omega} \times x) . & \\ \quad \nabla u+\vec{\omega} \times u+\nabla p=f & \text { in } \Omega, t>0, \\ \operatorname{div} u=0 & \text { in } \Omega, t>0, \\ u=\vec{\omega} \times x & \text { on } \Gamma, t>0, \\ u(x) \longrightarrow 0 & \text { as }|x| \longrightarrow+\infty, t>0 .\end{cases}
$$

where $\nu>0$ is the viscous coefficient, $f$ denotes the external forces. 
On the existence, uniqueness and regularity of solutions of (1), there are many papers, such as [1-8] and references therein. However, few results about the numerical analysis are developed. The reason is that the coefficient of " $\vec{\omega} \times x$ " tends to infinity as $|x| \longrightarrow+\infty$. A natural method to overcome this difficulty is the artificial boundary method. The main idea is that the exterior domain or the unbounded domain has to be truncated and the appropriate artificial boundary conditions are introduced. For example, Bonisch and Heuveline [9-11] study the adaptive finite element method for the free fall of a solid in a fluid occupied the whole space. Dunne and Rannacher $[12,13]$ study the adaptive finite element simulation for the fluid-structure interaction. On these references, we also refer the collected paper [14]. In [14], Bonisch et al. truncate the exterior domain to a bounded domain and introduce artificial "outflow" boundary conditions governed by the Gaussian heat kernel. They analyze the effect of the diameter of the truncated computational domain on the free-fall of the solid. To obtain the satisfactory accuracy, the diameter $d_{D}$ of the truncated domain must satisfy $d_{D} \geq 400$ if the length of the solid is $l=6$ and the width of the solid is $w=1$. Hence, for the artificial boundary method, the truncated domain must be sufficiently large. A direct effect to the numerical computation is adding the CPU time. The reason that why the truncated domain is sufficiently large is that the artificial "outflow" boundary conditions are not exact on the truncated boundary. To avoid using the large truncated domain, we study the coupled method of the boundary element and the finite element, which also is called the boundary integral method.

The boundary integral method is a good method to study the numerical method for the exterior problems or the unbounded problems. A general references can be found in [15]. The main idea is introducing some open ball which decomposes the exterior domain into a finite domain and a infinite domain, and transforming the equation in infinite domain to the boundary integral equation without using the artificial boundary condition. There are many papers about the boundary integral method for Navier-Stokes equations in exterior domain, such as [16-21] and references therein.

In this paper, we will apply the boundary integral method to the steady rotating Navier-Stokes equations in exterior domain. Firstly, we truncate the exterior domain by a open ball, and obtain a bounded domain and a new exterior domain which is outside the ball. In the bounded domain, we consider the rotating Navier-Stokes equations. But in the new exterior domain, we consider the Stokes problem. Subsequently, we transform the Stokes problem into a boundary integral equation on the boundary of the ball and avoid using the artificial boundary condition. It is noteworthiness that the boundary integral equation is exact on the boundary of the ball. Hence, we obtain the continuous formulation of the coupled method of the boundary element and the finite element. The main objective of this paper is to study the well-posedness of the continuous coupled problem (4) and show that the problem (4) admits a solution in a convex set. The finite element approximation of (4) and the numerical simulations will be given in the forthcoming papers. 


\section{Rotating Navier-Stokes equations}

Consider the following steady rotating Navier-Stokes equations:

$$
\begin{cases}-\nu \Delta u+u \cdot \nabla u+\vec{\omega} \times u-(\vec{\omega} \times x) \cdot \nabla u+\nabla p=f & \text { in } \Omega, \\ \operatorname{div} u=0 & \text { in } \Omega, \\ u=\vec{\omega} \times x & \text { on } \Gamma, \\ u(x) \longrightarrow 0 & \text { as } x \longrightarrow+\infty\end{cases}
$$

where $f \in L_{\text {loc }}^{2}(\Omega) \cap L_{\text {loc }}^{\frac{6}{5}}(\Omega)$.

Without loss of generality, we assume that the origin of coordinates lies in $\Omega^{c}$. Let $\delta\left(\Omega^{c}\right)$ be the coordinates of $\Omega^{c}$, that is

$$
\delta\left(\Omega^{c}\right)=\sup _{x, y \in \Omega^{c}}|x-y| .
$$

For each $R>\delta\left(\Omega^{c}\right)$, set

$$
\Omega_{R}=\Omega \cap B_{R}, \quad \Omega^{R}=\mathbb{R}^{3} \backslash \Omega_{R},
$$

where $B_{R}=\left\{x \in \mathbb{R}^{3}, \quad|x| \leq R\right\}$.

Firstly, we make the homogeneous of the boundary condition on $\Gamma$ in (2). Silvestre [4] showed the following lemma:

Lemma 2.1. For each $R>\delta\left(\Omega^{c}\right)$, there exists a smooth function $\psi \in C_{0}^{\infty}(\bar{\Omega})$ such that

$$
\left\{\begin{array}{l}
\operatorname{div} \psi=0 \quad \text { in } \Omega, \\
\psi=\vec{\omega} \times x \quad \text { on } \Gamma, \\
\|\psi\|_{q}+\|\nabla \psi\|_{r}+\left\|D^{2} \psi\right\|_{s} \leq \kappa_{1}|\vec{\omega}| \quad \text { for all } q>\frac{3}{2}, 1<r \leq 6,1 \leq s \leq 2, \\
\left|\int_{\Omega_{R}} u \cdot \nabla \psi \cdot u d x\right| \leq \frac{\nu}{2}\|\nabla u\|_{2, \Omega_{R}}^{2},
\end{array}\right.
$$

where $\kappa_{1}>0$ depends on $q, r, s, \nu, \Gamma$.

Let $u=v+\psi$ in (2), then $v$ satisfies

$$
\begin{cases}-\nu \Delta v+v \cdot \nabla v+v \cdot \nabla \psi+\psi \cdot \nabla v-(\vec{\omega} \times x) \cdot & \\ \nabla v+\vec{\omega} \times v+\nabla p=F & \Omega, \\ \operatorname{div} v=0 & \Omega, \\ v=0 & \Gamma, \\ v(x) \longrightarrow 0 & |x| \longrightarrow+\infty\end{cases}
$$

where

$$
F=\nu \Delta \psi+(\vec{\omega} \times x) \cdot \nabla \psi-\vec{\omega} \times \psi-\psi \cdot \nabla \psi+f
$$

According to Lemma 2.1, we can easily show $F \in L_{\text {loc }}^{2}(\Omega) \cap L_{\text {loc }}^{\frac{6}{5}}(\Omega)$ and

$$
\|F\|_{\frac{6}{5}} \leq \kappa_{2}|\vec{\omega}|
$$

where $\kappa_{2}>0$ depends on $\nu$ and $\Gamma$. 


\section{The boundary integral equation}

Select $R>\delta\left(\Omega^{c}\right)$ such that $\Omega_{R}$ contains the compact support of $f$ and $\psi$. The ball $B_{R}$ decomposes the unbounded domain $\Omega$ into a finite domain $\Omega_{R}$ and a exterior domain $\Omega^{R}$. We will use the following coupled problem to approximate the steady rotating Navier-Stokes equations (3):

$$
\begin{cases}-\nu \Delta v_{R}+v_{R} \cdot \nabla v_{R}+v_{R} \cdot \nabla \psi+\psi \cdot \nabla v_{R}+\vec{\omega} \times v_{R} \\ -(\vec{\omega} \times x) \cdot \nabla v_{R}+\nabla p_{R}=F & \text { in } \Omega_{R}, \\ \operatorname{div} v_{R}=0 & \text { in } \Omega_{R}, \\ -\nu \Delta v^{R}+\nabla p^{R}=0 & \text { in } \Omega^{R}, \\ \operatorname{div} v^{R}=0 & \text { in } \Omega^{R}, \\ v_{R}=0 & \text { on } \Gamma, \\ v_{R}=v^{R}, \lambda_{R}=\lambda^{R} & \text { on } \partial B_{R}\end{cases}
$$

where

$$
\begin{aligned}
& v_{R}=\left.v\right|_{\Omega_{R}}, v^{R}=\left.v\right|_{\Omega^{R}}, p_{R}=\left.p\right|_{\Omega_{R}}, p^{R}=\left.p\right|_{\Omega^{R}}, \\
& \lambda_{R}=\left(\left.\sum_{j=1}^{3} \sigma_{i j}\left(v_{R}, p_{R}\right) n_{j}\right|_{\partial B_{R}}\right)_{i}, \lambda^{R}=\left(\left.\sum_{j=1}^{3} \sigma_{i j}\left(v^{R}, p^{R}\right) n_{j}\right|_{\partial B_{R}}\right)_{i}, \\
& \sigma_{i j}\left(v_{R}, p_{R}\right)=-\delta_{i j} p_{R}+\nu\left(\frac{\partial v_{R i}}{\partial x_{j}}+\frac{\partial v_{R j}}{\partial x_{i}}\right) \\
& \sigma_{i j}\left(v^{R}, p^{R}\right)=-\delta_{i j} p^{R}+\nu\left(\frac{\partial v_{i}^{R}}{\partial x_{j}}+\frac{\partial v_{j}^{R}}{\partial x_{i}}\right)
\end{aligned}
$$

and $(v, p)$ is the solution of $(3)$. Moreover, $v^{R}(x) \longrightarrow 0$ as $|x| \longrightarrow+\infty$.

Let $\left(E_{i j}(x, y), P_{i}(x, y)\right)(i, j=1,2,3)$ be the fundamental solutions of three dimensional Stokes problem, then they satisfy

$$
\left\{\begin{array}{l}
-\nu \Delta E_{i j}(x-y)+\frac{\partial}{\partial x_{j}} P_{i}(x-y)=\delta_{i j} \delta(x-y), \\
\frac{\partial}{\partial x_{i}} E_{i j}(x-y)=0
\end{array}\right.
$$

where $\delta_{i j}$ is Kronecker symbol and $\delta(x-y)$ is the Dirac function. It is well known that $\left(E_{i j}(x, y), P_{i}(x, y)\right)$ have the following forms

$$
\left\{\begin{array}{l}
E_{i j}(x, y)=-\frac{1}{8 \pi}\left(\frac{\delta_{i j}}{|x-y|}+\frac{\left(x_{i}-y_{i}\right)\left(x_{j}-y_{j}\right)}{|x-y|^{3}}\right) \\
P_{i}(x, y)=\frac{1}{4 \pi} \frac{x_{i}-y_{i}}{|x-y|^{3}} .
\end{array}\right.
$$


Ladyzhenskaya [22] shows that if $(v, p)$ is a solution of (4) in $\Omega^{R}$, then for $x \in \Omega^{R}$, there holds

$$
\left\{\begin{aligned}
v_{k}(x)= & -\int_{\partial B_{R}} v(y) \cdot \sigma\left(E_{k}, P_{k}\right)(x-y) \cdot n(y) \mathrm{d} s(y) \\
& +\int_{\partial B_{R}} E_{k}(x-y) \cdot \lambda(y) \mathrm{d} s(y) \quad k=1,2,3 \\
p(x)= & -2 \nu \int_{\partial B_{R}} \frac{\partial P}{\partial n}(x-y) \cdot v(y) \mathrm{d} s(y) \\
& +\int_{\partial B_{R}} P(x-y) \cdot \lambda(y) \mathrm{d} s(y)
\end{aligned}\right.
$$

where

$$
\left\{\begin{array}{l}
E_{k}(x-y)=\left(E_{k 1}(x-y), E_{k 2}(x-y), E_{k 3}(x-y)\right) \\
P(x-y)=\left(P_{1}(x-y), P_{2}(x-y), P_{3}(x-y)\right) \\
\sigma_{i j}(v, p)=-\delta_{i j} p+\nu\left(\frac{\partial v_{i}}{\partial x_{j}}+\frac{\partial v_{j}}{\partial x_{i}}\right) \\
\lambda=\left(\left.\sum_{j=1}^{3} \sigma_{i j}(v, p) n_{j}\right|_{\partial B_{R}}\right)_{i}, \quad i=1,2,3
\end{array}\right.
$$

Moreover, if $x \in \partial B_{R}$, there holds

$$
\begin{aligned}
\frac{1}{2} v_{k}(x)= & -\int_{\partial B_{R}} v(y) \cdot \sigma\left(E_{k}, P_{k}\right)(x-y) \cdot n(y) \mathrm{d} s(y) \\
& +\int_{\partial B_{R}} E_{k}(x-y) \cdot \lambda(y) \mathrm{d} s(y) \quad k=1,2,3,
\end{aligned}
$$

which is the boundary integral equation.

\section{The coupled problem}

If $(v, p)$ is the solution of $(4)$ in $\Omega_{R}$, then

$$
\begin{cases}-\nu \Delta v+v \cdot \nabla v+v \cdot \nabla \psi+\psi \cdot \nabla v+\vec{\omega} \times v-(\vec{\omega} \times x) \cdot & \\ \quad \nabla v+\nabla p=F & \text { in } \Omega_{R} \\ \operatorname{div} v=0 & \text { in } \Omega_{R} \\ v=0 & \text { on } \Gamma .\end{cases}
$$

Introduce the Hilbert space

$$
V=\left\{v \in H^{1}\left(\Omega_{R}\right)^{3}, \quad v=0 \text { on } \Gamma\right\}
$$

with norm

$$
\|\nabla v\|=\left(\int_{\Omega_{R}}|\nabla v|^{2} \mathrm{~d} x\right)^{\frac{1}{2}} .
$$

Denote by $V_{\sigma}$ the solenoidal subspace of $V$ and

$$
M=\left\{q \in L^{2}\left(\Omega_{R}\right), \int_{\Omega_{R}} q \mathrm{~d} x=0\right\}=L_{0}^{2}\left(\Omega_{R}\right)
$$


with $L^{2}\left(\Omega_{R}\right)$-norm. Introduce the following bilinear forms and trilinear form:

$$
\left\{\begin{array}{l}
a(u, v)=2 \nu \sum_{i, j=1}^{3} \int_{\Omega_{R}} \varepsilon_{i j}(u) \varepsilon_{i j}(v) \mathrm{d} x \\
d(v, p)=\sum_{i=1}^{3} \int_{\Omega_{R}} p \frac{\partial v_{i}}{\partial x_{i}} \mathrm{~d} x \\
b(u, v, w)=\sum_{i, j=1}^{3} \int_{\Omega_{R}} u_{i} \frac{\partial v_{j}}{\partial x_{i}} w_{j} \mathrm{~d} x \\
(f, v)=\sum_{i=1}^{3} \int_{\Omega_{R}}^{3} f_{i} v_{i} \mathrm{~d} x \\
<v, \lambda>=\sum_{i=1}^{3} \int_{\partial B_{R}} \lambda_{i} v_{i} \mathrm{~d} s(x)
\end{array}\right.
$$

where $\langle\cdot, \cdot\rangle$ denotes the duality pairing between $H^{-\frac{1}{2}}\left(\partial B_{R}\right)^{3}$ and $H^{\frac{1}{2}}\left(\partial B_{R}\right)^{3}, \varepsilon_{i j}(u)=\frac{1}{2}\left(\frac{\partial u_{i}}{\partial x_{j}}+\frac{\partial u_{j}}{\partial x_{i}}\right)$. Then the variational formulation of $(6)$ is

$$
\left\{\begin{array}{c}
a(v, w)+b(v, v, w)+b(v, \psi, w)+b(\psi, v, w)-b(\vec{\omega} \times x, v, w) \\
+(\vec{\omega} \times v, w)-d(w, p)+<w, \lambda>=(F, w) \quad \forall w \in V, \\
d(v, q)=0 \quad \forall q \in M .
\end{array}\right.
$$

It is well known that (7) is equivalent to

$$
\begin{gathered}
a(v, w)+b(v, v, w)+b(v, \psi, w)+b(\psi, v, w)-b(\vec{\omega} \times x, v, w) \\
+(\vec{\omega} \times v, w)+<w, \lambda>=(F, w) \quad \forall w \in V_{\sigma} .
\end{gathered}
$$

On the other hand, if $(v, p)$ is the solution of (4) in $\Omega^{R}$, then

$$
\begin{cases}-\nu \Delta v+\nabla p=0 & \text { in } \Omega^{R}, \\ \operatorname{div} v=0 & \text { in } \Omega^{R}, \\ v(x) \longrightarrow 0 & \text { as } x \longrightarrow+\infty .\end{cases}
$$

Denote

$$
T=\left\{\mu \in H^{-\frac{1}{2}}\left(\partial B_{R}\right)^{3}, \quad \int_{\partial B_{R}} \mu \mathrm{d} s=0\right\}
$$

Then

$$
\lambda=\left(\left.\sum_{j=1}^{3} \sigma_{i j}(v, p) n_{j}\right|_{\partial B_{R}}\right)_{i} \in T, \quad i=1,2,3 .
$$

In fact,

$$
\begin{aligned}
\int_{\partial B_{R}} \lambda \mathrm{d} s & =\int_{\partial B_{R}} \sigma(v, p) \cdot n \mathrm{~d} s \\
& =\int_{\Omega^{R}} \operatorname{div} \sigma(v, p) \mathrm{d} x \\
& =\int_{\Omega^{R}}(\nu \Delta v-\nabla p) \mathrm{d} x=0 .
\end{aligned}
$$


Multiplying (5) by $\mu \in T$ and integrating over $\partial B_{R}$ yields

$$
2 b(\lambda, \mu)-<v, \mu>-2<G v, \mu>=0 \quad \forall \mu \in T,
$$

where $G=\left\{G_{1}, G_{2}, G_{3}\right\}$, and

$$
\left\{\begin{array}{l}
b(\lambda, \mu)=\sum_{i, k=1}^{3} \int_{\partial B_{R}} \int_{\partial B_{R}} \mu_{k}(x) E_{k i}(x-y) \lambda_{i}(y) \mathrm{d} s(y) \mathrm{d} s(x), \\
G_{k}(v)=\int_{\partial B_{R}} v(y) \cdot \sigma\left(E_{k}, P_{k}\right)(x-y) \cdot n(y) \mathrm{d} s(y) .
\end{array}\right.
$$

Thus, the variational formulation of the coupled problem by the rotating Navier-Stokes equations (6) in finite domain $\Omega_{R}$ and the boundary integral equation (5) is

$$
\left\{\begin{array}{l}
\text { Find }(v, \lambda, p) \in V \times T \times M \text { such that } \\
a(v, w)+b(v, v, w)+b(v, \psi, w)+b(\psi, v, w)-b(\vec{\omega} \times x, v, w) \\
\quad+(\vec{\omega} \times v, w)-d(w, p)+<w, \lambda>=(F, w) \quad \forall w \in V, \\
2 b(\lambda, \mu)-<v, \mu>-2<G v, \mu>=0 \quad \forall \mu \in T, \\
d(v, q)=0 \quad \forall q \in M .
\end{array}\right.
$$

Moreover, the variational formulation (10) is equivalent to

$$
\left\{\begin{array}{l}
\text { Find }(v, \lambda) \in V_{\sigma} \times T \text { such that } \\
a(v, w)+b(v, v, w)+b(v, \psi, w)+b(\psi, v, w)-b(\vec{\omega} \times x, v, w) \\
\quad+(\vec{\omega} \times v, w)+<w, \lambda>=(F, w) \quad \forall w \in V_{\sigma}, \\
2 b(\lambda, \mu)-<v, \mu>-2<G v, \mu>=0 \quad \forall \mu \in T .
\end{array}\right.
$$

Observe that the trilinear term satisfies

$$
b(u, v, w) \leq c_{0} R\|u\|_{V}\|w\|_{V}\|w\|_{V},
$$

where $c_{0}>0$ is independent of $R$. The coefficient $c_{0} R$ brings the difficulty to show the existence of solution of the problem (11). In this case, we can show that the solution exists only if $\nu>c R$, where $c>0$ is independent of $R$. However, the condition $\nu>c R$ is too strict for the fluids. To avoid using this condition, we will consider the problem (6) in a closed convex subset of $V$. Denote by $K$ the closed convex subset of $\mathrm{V}$

$$
K=\left\{v \in V, \quad\|v \cdot n\|_{2, \partial B_{R}} \leq \kappa_{2}\right\},
$$

where $\kappa_{2}>0$ is a positive constant. Then the variational formulation of the coupled problem is the following variational inequality:

$$
\left\{\begin{array}{l}
\text { Find }(v, \lambda, p) \in K \times T \times M \text { such that } \\
a(v, w-v)+b(v, v, w-v)+b(v, \psi, w-v)+b(\psi, v, w-v) \\
\quad-b(\vec{\omega} \times x, v, w-v)+(\vec{\omega} \times v, w-v)-d(w-v, p) \\
\quad+<w-v, \lambda>\geq(F, w-v) \quad \forall w \in K, \\
2 b(\lambda, \mu)-<v, \mu>-2<G v, \mu>=0 \quad \forall \mu \in T, \\
d(v, q)=0 \quad \forall q \in M .
\end{array}\right.
$$


Denote by $K_{\sigma}$ the solenoidal subset of $K$, then (12) is equivalent to

$$
\left\{\begin{array}{l}
\text { Find }(v, \lambda) \in K_{\sigma} \times T \text { such that } \\
a(v, w-v)+b(v, v, w-v)+b(v, \psi, w-v)+b(\psi, v, w-v) \\
\quad-b(\vec{\omega} \times x, v, w-v)+(\vec{\omega} \times v, w-v) \\
\quad+<w-v, \lambda>\geq(F, w-v) \quad \forall w \in K_{\sigma}, \\
2 b(\lambda, \mu)-<v, \mu>-2<G v, \mu>=0 \quad \forall \mu \in T .
\end{array}\right.
$$

\section{Existence of solution}

In this section, we will show the existence of (13) by the Galerkin method in [23]. Firstly, we introduce the following lemma due to Kaitai Li and Yinnian He $[16]$ :

Lemma 5.1. The bilinear form $b(\cdot, \cdot)$ is symmetric and continuous on $T \times T$. Moreover, there exists a positive constant $c>0$ such that

$$
b(\mu, \mu) \geq c\|\mu\|_{H^{-\frac{1}{2}}\left(\partial B_{R}\right)^{3}}^{2} \quad \forall \mu \in T .
$$

Then for given $v \in V_{\sigma}$, the problem

$$
\left\{\begin{array}{l}
\text { Find } \lambda \in T \text { such that } \\
2 b(\lambda, \mu)-<v, \mu>-2<G v, \mu>=0 \quad \forall \mu \in T
\end{array}\right.
$$

admits a unique solution $\lambda=\lambda(v)$. Hence, the problem (13) is equivalent to

$$
\left\{\begin{array}{l}
\text { Find } v \in K_{\sigma} \text { such that } \\
a(v, w-v)+b(v, v, w-v)+b(v, \psi, w-v)+b(\psi, v, w-v) \\
\quad-b(\vec{\omega} \times x, v, w-v)+(\vec{\omega} \times v, w-v)+<w-v, \lambda(v)> \\
\geq(F, w-v) \quad \forall w \in K_{\sigma} .
\end{array}\right.
$$

On $\lambda(v)$, we have

Lemma 5.2. For given $v \in V_{\sigma}$, there holds

$$
<v, \lambda(v)>\geq 0 \text {. }
$$

Proof. Consider the following problem

$$
\left\{\begin{array}{lr}
-\nu \Delta w+\nabla \pi=0 & \text { in } \Omega_{R} \\
\operatorname{div} w=0 & \text { in } \Omega_{R}, \\
\left.w\right|_{\Gamma}=0,\left.\quad w\right|_{\partial B_{R}}=\left.v\right|_{\partial B_{R} .} &
\end{array}\right.
$$

It is easy to show that the problem (14) exists a unique solution $w \in V_{\sigma}$. Then

$$
\begin{aligned}
<v, \lambda(v)>= & \int_{\partial B_{R}} \sigma^{i j}(v, p) n_{i} v_{j} \mathrm{~d} s=\int_{\partial B_{R}} \sigma^{i j}(w, \pi) n_{i} w_{j} \mathrm{~d} s \\
= & \int_{\Omega_{R}} \operatorname{div}(\sigma(w, \pi) \cdot w) \mathrm{d} x=\int_{\Omega_{R}} \operatorname{div} \sigma(w, \pi) \cdot w \mathrm{~d} x \\
& +\int_{\Omega_{R}} \sigma^{i j}(w, \pi) \varepsilon^{i j}(w) \mathrm{d} x \\
= & \int_{\Omega_{R}} \sigma^{i j}(w, \pi) \varepsilon^{i j}(w) \mathrm{d} x=2 \nu \int_{\Omega_{R}} \varepsilon^{i j}(w) \varepsilon^{i j}(w) \mathrm{d} x \geq 0
\end{aligned}
$$


Define the projection operator from $V_{\sigma}$ to $K_{\sigma}$. For each $u \in V_{\sigma}$, let $\Phi(u)=u-P u$. Introduce the following lemmas:

Lemma 5.3. [24] For all $v \in V_{\sigma}$, there exists a unique $P v \in K_{\sigma}$ such that

$$
\begin{cases}(v-P v, w-P v)_{1} \leq 0 & \forall w \in K_{\sigma}, \\ \left\|P v_{1}-P v_{2}\right\|_{V} \leq\left\|v_{2}-v_{1}\right\|_{V} & \forall v_{1}, v_{2} \in V_{\sigma},\end{cases}
$$

where $(\cdot, \cdot)_{1}$ is the inner product in $V$.

Lemma 5.4. [23] For all $v \in V_{\sigma}$, there holds

$$
(\Phi(v), v)_{1} \geq\|\Phi(v)\|_{V}^{2} .
$$

Since $V_{\sigma}$ is divisible and reflexive, there exists a standard orthogonal basis $\left\{\phi_{i}\right\}_{i=1}^{\infty}$ which is dense and complete in $V_{\sigma}$. Denote by $V_{n}=\left\{\phi_{1}, \ldots, \phi_{n}\right\}$, then we define the Galerkin approximation solution of (15) $v^{n}=\sum_{k=1}^{n} \alpha_{k} \phi_{k} \in V_{n}$ by

$$
\begin{aligned}
& a\left(v^{n}, \phi_{k}\right)+b\left(P v^{n}, v^{n}, \phi_{k}\right)+b\left(v^{n}, \psi, \phi_{k}\right)+b\left(\psi, v^{n}, \phi_{k}\right)-b\left(\vec{\omega} \times x, v^{n}, \phi_{k}\right) \\
& \quad+\left(\vec{\omega} \times v^{n}, \phi_{k}\right)+<\phi_{k}, \lambda\left(v^{n}\right)>+n\left(\Phi\left(v^{n}\right), \phi_{k}\right)_{1}=\left(F, \phi_{k}\right),
\end{aligned}
$$

where $k=1, \ldots, n$. Multiplying $\alpha_{k}$ in (17) and summing for $k=1, \ldots, n$, we obtain

$$
\begin{aligned}
& a\left(v^{n}, v^{n}\right)+b\left(P v^{n}, v^{n}, v^{n}\right)+b\left(v^{n}, \psi, v^{n}\right)+b\left(\psi, v^{n}, v^{n}\right)-b\left(\vec{\omega} \times x, v^{n}, v^{n}\right) \\
& \quad+\left(\vec{\omega} \times v^{n}, v^{n}\right)+<v^{n}, \lambda\left(v^{n}\right)>+n\left(\Phi\left(v^{n}\right), v^{n}\right)_{1}=\left(F, v^{n}\right) .
\end{aligned}
$$

From Lemma 5.4, one has

$$
a\left(v^{n}, v^{n}\right)+b\left(P v^{n}, v^{n}, v^{n}\right)+b\left(v^{n}, \psi, v^{n}\right) \leq\left(F, v^{n}\right),
$$

where we use

$$
b\left(\psi, v^{n}, v^{n}\right)=b\left(\vec{\omega} \times x, v^{n}, v^{n}\right)=0, \quad\left(\vec{\omega} \times v^{n}, v^{n}\right)=0
$$

and

$$
<v^{n}, \lambda\left(v^{n}\right)>\geq 0, \quad\left(\Phi\left(v^{n}\right), v^{n}\right)_{1} \geq 0 .
$$

In terms of Lemma 2.1, one has

$$
\begin{aligned}
& a\left(v^{n}, v^{n}\right)+b\left(P v^{n}, v^{n}, v^{n}\right)+b\left(v^{n}, \Psi, v^{n}\right) \\
& \geq \nu\left\|v^{n}\right\|_{V}^{2}-\frac{\nu}{2}\left\|v^{n}\right\|_{V}^{2}-\left.\frac{1}{2}\left|\int_{\partial B_{R}} P v^{n} \cdot n\right| v^{n}\right|^{2} \mathrm{~d} s \mid \\
& \geq \frac{\nu}{2}\left\|v^{n}\right\|_{V}^{2}-\frac{\kappa_{2} c_{1}}{2}\left\|v^{n}\right\|_{V}^{2}=\frac{\nu-\kappa_{2} c_{1}}{2}\left\|v^{n}\right\|_{V}^{2} .
\end{aligned}
$$

Assume $\nu>\kappa_{2} c_{1}$, then

$$
\frac{\nu-\kappa_{2} c_{1}}{2}\left\|v^{n}\right\|_{V}^{2} \leq\|F\|_{\frac{6}{5}}\left\|v^{n}\right\|_{V} \leq \kappa_{1}\left|\vec{\omega}\left\|\mid v^{n}\right\|_{V} .\right.
$$

That is

$$
\left\|v^{n}\right\|_{V} \leq \frac{2 \kappa_{1}}{\nu-\kappa_{2} c_{1}}|\vec{\omega}|
$$


From Lemma 5.4, we have

$$
\begin{aligned}
n\left\|\Phi\left(v^{n}\right)\right\|_{V}^{2} & \leq n\left(\Phi\left(v^{n}\right), v^{n}\right) \\
& \leq\left(F, v^{n}\right)-a\left(v^{n}, v^{n}\right)-b\left(v^{n}, \psi, v^{n}\right)-b\left(P v^{n}, v^{n}, v^{n}\right) \\
& \leq\|F\|_{\frac{6}{5}}\left\|v^{n}\right\|_{V}+\frac{3 \nu}{2}\left\|v^{n}\right\|_{V}^{2}+\frac{\kappa_{2} c_{1}}{2}\left\|v^{n}\right\|_{V}^{2},
\end{aligned}
$$

which together (18) show

$$
\lim _{n \rightarrow+\infty}\left\|\Phi\left(v^{n}\right)\right\|_{V} \longrightarrow 0 .
$$

Since $v^{n}$ is uniformly bounded in $V_{\sigma}$, there exist a subsequence of $\left\{v^{n}\right\}$, which is denoted by $\left\{v^{n}\right\}$ again, and a $v \in V_{\sigma}$ satisfying (18) such that $v^{n}$ converges weakly to $v$ in $V$ and converges strongly to $v$ in $L^{2}\left(\Omega_{R}\right)^{3}$ and $L^{p}\left(\partial B_{R}\right)^{3}$ for $2 \leq p<4$. With the similar method in [23], we have $v \in K_{\sigma}$ and $P v=v$. For each $w^{m}=\sum_{k=1}^{m} \beta_{k} \phi_{k} \in K_{\sigma}, m<n$ and $v^{n}$, we have

$$
\begin{aligned}
& a\left(v^{n}, w^{m}-P v^{n}\right)+b\left(P v^{n}, v^{n}, w^{m}-P v^{n}\right)+b\left(v^{n}, \psi, w^{m}-P v^{n}\right) \\
& \quad+b\left(\psi, v^{n}, w^{m}-P v^{n}\right)-b\left(\vec{\omega} \times x, v^{n}, w^{m}-P v^{n}\right)+\left(\vec{\omega} \times v^{n}, w^{m}-P v^{n}\right) \\
& \quad+<w^{m}-P v^{n}, \lambda\left(v^{n}\right)>+n\left(\Phi\left(v^{n}\right), w^{m}-P v^{n}\right)=\left(F, w^{m}-P v^{n}\right) .
\end{aligned}
$$

From Lemma 5.3, there holds

$$
\left(\Phi\left(v^{n}\right), w^{m}-P v^{n}\right)=\left(v^{n}-P v^{n}, w^{m}-P v^{n}\right) \leq 0 .
$$

Hence,

$$
\begin{aligned}
& a\left(v^{n}, w^{m}-P v^{n}\right)+b\left(P v^{n}, v^{n}, w^{m}-P v^{n}\right)+b\left(v^{n}, \psi, w^{m}-P v^{n}\right) \\
& \quad+b\left(\psi, v^{n}, w^{m}-P v^{n}\right)-b\left(\vec{\omega} \times x, v^{n}, w^{m}-P v^{n}\right)+\left(\vec{\omega} \times v^{n}, w^{m}-P v^{n}\right) \\
& \quad+<w^{m}-P v^{n}, \lambda\left(v^{n}\right)>\geq\left(F, w^{m}-P v^{n}\right) .
\end{aligned}
$$

Next, we will show the following relations:

$$
\begin{gathered}
\lim _{n \rightarrow+\infty} b\left(P v^{n}, v^{n}, P v^{n}\right)=b(v, v, v), \\
\lim _{n \rightarrow+\infty} b\left(v^{n}, \psi, P v^{n}\right)=b(v, \psi, v), \\
\lim _{n \rightarrow+\infty} b\left(\psi, v^{n}, P v^{n}\right)=b(\psi, v, v), \\
\lim _{n \rightarrow+\infty} b\left(\psi, v^{n}, w^{m}\right)=b\left(\psi, v, w^{m}\right), \\
\lim _{n \rightarrow+\infty}<P v^{n}, \lambda\left(v^{n}\right)>=<v, \lambda(v)>.
\end{gathered}
$$

About (20), we have

$$
\begin{aligned}
& \left|b\left(P v^{n}, v^{n}, P v^{n}\right)-b(v, v, v)\right| \\
& \quad \leq\left|b\left(P v^{n}, v^{n}, v^{n}\right)-b(v, v, v)\right|+\left|b\left(P v^{n}, v^{n}, \Phi\left(v^{n}\right)\right)\right|:=I_{1}+I_{2} .
\end{aligned}
$$

Since $\Phi\left(v^{n}\right)$ converges to 0 in $V$ as $n \rightarrow+\infty$, it is easy to show

$$
I_{2} \leq c\left\|P v^{n}\right\|_{V}\left\|v^{n}\right\|_{V}\left\|\Phi\left(v^{n}\right)\right\|_{V} \longrightarrow 0 \quad \text { as } n \rightarrow+\infty .
$$


For $I_{1}$, we have

$$
\begin{aligned}
I_{1} & =\left.\frac{1}{2}\left|\int_{\partial B_{R}} P v^{n} \cdot n\right| v^{n}\right|^{2}-v \cdot n\left|v^{2}\right| \mathrm{d} s \mid \\
& \leq \frac{1}{2}\left|\int_{\partial B_{R}} P v^{n} \cdot n\left(\left|v^{n}\right|^{2}-|v|^{2}\right) \mathrm{d} s\right|+\left.\frac{1}{2}\left|\int_{\partial B_{R}}\left(P v^{n} \cdot n-v \cdot n\right)\right| v\right|^{2} \mathrm{~d} s \mid \\
& \leq c|| P v^{n} \cdot n\left\|_{\alpha, \partial B_{R}}\left(\left\|v^{n}\right\|_{\alpha^{\prime}, \partial B_{R}}^{2}-\|v\|_{\alpha^{\prime}, \partial B_{R}}^{2}\right)+c|| v^{n}-v\right\|_{2, \partial B_{R}}\|v\|_{4, \partial B_{R}}^{2} \\
& \longrightarrow 0 \text { as } n \rightarrow+\infty,
\end{aligned}
$$

where $2<\alpha \leq 4$ and $\frac{8}{3} \leq \alpha^{\prime}=\frac{2 \alpha}{\alpha-1}<4$. We complete the proof of (20). Next, we show $(21)$.

$$
\begin{aligned}
& \left|b\left(v^{n}, \psi, P v^{n}\right)-b(v, \psi, v)\right| \\
& \quad \leq\left|b\left(v^{n}, \psi, v^{n}\right)-b(v, \psi, v)\right|+\left|b\left(v^{n}, \psi, \Phi\left(v^{n}\right)\right)\right| \\
& \quad \leq\left|b\left(v^{n}-v, \psi, v^{n}\right)\right|+\left|b\left(v, \psi, v^{n}-v\right)\right|+\left|v\left(v^{n}, \psi, \Phi\left(v^{n}\right)\right)\right| \\
& \quad \leq\left. c\left\|v^{n}-v\right\|\right|^{\frac{1}{2}}\left\|v^{n}-v\right\|_{V}^{\frac{1}{2}}\|\psi\|\left\|_{V}\right\| v^{n}\left\|_{V}+c\right\| v^{n}\left\|_{V}\right\| \psi\left\|_{V}\right\| \Phi\left(v^{n}\right) \|_{V} \\
& \quad \longrightarrow 0 \quad \text { as } n \rightarrow+\infty .
\end{aligned}
$$

For (22), we have

$$
\begin{aligned}
& \left|b\left(\psi, v^{n}, P v^{n}\right)-b(\psi, v, v)\right| \\
& \quad \leq\left|b\left(\psi, v^{n}, v^{n}\right)-b(\psi, v, v)\right|+\left|b\left(\psi, v^{n}, \Phi\left(v^{n}\right)\right)\right| \\
& \quad=\left|b\left(\psi, v^{n}, \Phi\left(v^{n}\right)\right)\right| \leq c\|\psi\|_{V}\left\|v^{n}\right\|_{V}|| \Phi\left(v^{n}\right) \|_{V} \longrightarrow 0 \quad \text { as } n \rightarrow+\infty .
\end{aligned}
$$

For (23), we have

$$
\begin{aligned}
& \left|b\left(\psi, v^{n}, w^{m}\right)-b\left(\psi, v, w^{m}\right)\right| \\
& \quad \leq\left|b\left(\psi, w^{m}, v^{n}\right)-b\left(\psi, w^{m}, v\right)\right| \\
& \quad \leq c\|\psi\|_{V}\left\|w^{m}\right\|_{V}\left\|v-v^{n}\right\|^{\frac{1}{2}}\left\|v-v^{n}\right\|_{V}^{\frac{1}{2}} \\
& \quad \longrightarrow 0 \text { as } n \rightarrow+\infty .
\end{aligned}
$$

For (24), we have

$$
\begin{aligned}
\mid< & P v^{n}, \lambda\left(v^{n}\right)>-<v, \lambda(v)>\mid \\
& \leq\left|<v^{n}, \lambda\left(v^{n}\right)>-<v, \lambda(v)>\right|+\left|<\Phi\left(v^{n}\right), \lambda\left(v^{n}\right)>\right| \\
& \leq\left|<v^{n}-v, \lambda\left(v^{n}\right)>\right|+\left|<v, \lambda\left(v^{n}-v\right)>\right|+\left|<\Phi\left(v^{n}\right), \lambda\left(v^{n}\right)>\right| \\
& =I_{3}+I_{4}+I_{5} .
\end{aligned}
$$

We can easily show

$$
I_{3}, I_{5} \longrightarrow 0 \quad \text { as } n \rightarrow+\infty .
$$

Since $\lambda$ is continuous, then $\lambda\left(v^{n}\right)$ converges to $\lambda(v)$ in $L^{2}\left(\partial B_{R}\right)^{3}$ and

$$
I_{4} \longrightarrow 0 \quad \text { as } n \rightarrow+\infty .
$$

Hence, we obtain (24). Hence, pass to limit $n \rightarrow+\infty$ in (19), we have

$$
\begin{aligned}
& a\left(v, w^{m}-v\right)+b\left(v, v, w^{m}-v\right)+b\left(v, \psi, w^{m}-v\right)+b\left(\psi, v, w^{m}-v\right) \\
& \quad-b\left(\vec{\omega} \times x, v, w^{m}-v\right)+\left(\vec{\omega} \times v, w^{m}-v\right)+<w^{m}-v, \lambda(v)>\geq\left(F, w^{m}-v\right) .
\end{aligned}
$$


According to the property of $\phi_{k}$, For all $w \in K_{\sigma}$, The variational inequality (15) holds. Thus, we show the following theorem:

Theorem 5.1. If for sufficiently large $\nu$ such that $\nu>\kappa_{2} c_{1}$, then the coupled problem (13) exists a solution $(v, \lambda) \in K_{\sigma} \times T$, where $c_{1}>0$ is independent of $R$, and $v$ satisfies

$$
\|v\|_{V} \leq \frac{2 \kappa_{3}}{\nu-\kappa_{4} c_{1}}|\vec{\omega}|
$$

\section{Acknowledgements}

The authors would like to thank the reviewer for pointing out the Ref. [14] to the authors and for giving some valuable advice to improve this manuscript.

\section{References}

[1] Galdi, G.P., Silvestre, A.L.: Strong solution to the Navier-Stokes equations around a rotating obstacle. Arch. Ration. Mech. Anal. 176(3), 331-350 (2005)

[2] Galdi, G.P.: On the motion of a rigid body in a viscous liquid: a mathematical analysis with applications. In: Handbook of Mathematical Fluid Dynamics, pp. 653-791. Elsevier Science, Amsterdam (2002)

[3] Galdi, G.P.: Steady flow of a Navier-Stokes fluid around a rotating obstacle. J. Elast. 71(1), 1-31 (2003)

[4] Silvestre, A.L.: On the existence of steady flows of a Navier-Stokes liquid around a moving rigid body. Math. Methods Appl. Sci. 27(12), 1399-1409 (2004)

[5] Geissert, M., Hech, H., Hieber, M.: $L^{p}$-theory of the Navier-Stokes flow in the exterior of a moving or rotating obstacle. J. Reine Angew. Math. 596(1), 45-62 (2006)

[6] Hishida, T.: $L^{q}$ estimates of weak solutions to the stationary Stokes equations around a rotating body. J. Math. Soc. 58(3), 742-767 (2006)

[7] Hishida, T.: An existence theorem for the Navier-Stokes flow in the exterior of a rotating obstacle. Arch. Ration. Mech. Anal. 150(1999), 307-348 (1999)

[8] Farwig, R.: An $L^{q}$-analysis of viscous fluid flow past a rotating obstacle. Tohoku Math. J. 58(1), 129-147 (2006)

[9] Bonish, S.: Adaptive finite-element mehthods for rigid particulate flow problems. Doctoral thesis, Institute of Applied Mathematics, University of Heidelberg (2006)

[10] Bonish, S., Heuveline, V.: On the numerical simulation of the instationary free fall of a solid in a fluid. I. The Newtonian case. Comput. Fluids 36, 1434-1445 (2007) 
[11] Bonish, S., Heuveline, V.: On the numerical simulation of the instationary free fall of a solid in a fluid. II. The viscoelastic case. SFB Preprint 2004-32, University of Heidelberg (2004)

[12] Dunne, T.: Adaptive finite element simulation of fluid structure interaction based on an Eulerian formulation. Doctoral thesis, Institute of Applied Mathematics, University of Heidelberg (2007)

[13] Dunne, T., Rannacher, R.: Adaptive finite element approximation of fluid-structure interaction based on an Eulerian variational formulation. In: Bungartz, H.J., Schafer, M. (eds.) Fluid-Structure Interaction: Modelling, Simulation, Optimisation. Springer's LNCSE-Series (2006)

[14] Bonisch, S., Dunne, T., Rannacher, R.: Numerics of fluid-structure interaction. Hemodynamical flows. Modeling, analysis and simulation. Oberwolfach Seminars, 37, pp. 333-378 (2008)

[15] Yu, D.: Natural Boundary Integral Method and its Applications. Science Press, Beijing (2002)

[16] Li, K., He, Y.: The coupling of boundary integral and finite element methods for the Navier-Stokes in an exterior domain. J. Comput. Math. 7(2), 157-173 (1989)

[17] Li, K., He, Y.: Coupling method for the exterior stationary Navier-Stokes equations. Numer. Methods Partial Differ. Equ. 9(1), 35-49 (1993)

[18] He, Y., Li, K.: The coupling of boundary integral and finite element methods for the nonstationary exterior flow. Numer. Methods Partial Differ. Equ. 14(5), 549-565 (1998)

[19] He, Y., Li, K.: Stokes coupling method for the exterior flow part I: approximate accuracy. Acta Math. Appl. Sin. 15(3), 333-336 (1999)

[20] He, Y., Li, K.: The coupling method of finite elements and boundary elements for radiation problem. Acta Math. Appl. Sin. 6(2), 97-112 (1990)

[21] He, Y., Li, K.: Stokes coupling method for the exterior flow Part III: regularity. Prog. Nat. Sci. 11(10), 734-745 (2001)

[22] Ladyzhenskaya, O.A.: The Mathematical Theory of Viscous Incompressible Flow. Gordon and Breach, London (1969)

[23] Kracmar, S., Neustupa, J.: A Weak Solvability of a Steady Variational Inequality of the Navier-Stokes Type with Mixed Boundary Conditions. Nonlinear Anal. 47(6), 4169-4180 (2001)

[24] Kinderlehrer, D., Stampacchia, G.: An Introduction to Variational Inequalities and Their Applications. SIAM, Philadelphia (2000)

R. An

College of Mathematics and Information Science,

Wenzhou University,

325035 Wenzhou, China

e-mail: anrong702@yahoo.com.cn 
K. Li

School of Science,

Xi'an Jiaotong University,

710049, Xi'an, China

Received: 30 October 2008.

Accepted: 31 August 2009. 